\title{
Transtibial prosthetic socket pistoning: Static evaluation of Seal-In® X5 and Dermo® Liner using motion analysis system
}

\author{
H. Gholizadeh a,, N.A. Abu Osman a, M. Kamyab b, A. Eshraghi a, W.A.B. Wan Abas a, M.N. Azam c \\ a Department of Biomedical Engineering, Faculty of Engineering, University of Malaya, Malaysia \\ b Department of Orthotics and Prosthetics, Faculty of Rehabilitation Science, Tehran University of Medical Sciences, Tehran, Iran \\ c Department of Applied Statistic, Faculty of Economics and Administration, University of Malaya, Malaysia \\ Introduction
}

The lower limb prosthesis's efficiency is mainly guaranteed by its

optimal suspension method in order to secure the socket to the

amputee's stump. In fact, suspension and fitting play the main role in

comfort and prosthetic function (Baars and Geertzen, 2005; Isozaki

et al., 2006; Kristinsson, 1993; Tanner and Berke, 2001).

In addition, themost important factormentioned by the amputees is

the fit of their prosthesis and suspension (Datta et al., 1996; Fillauer

et al., 1989; Legro et al., 1999). In some studies regarding lower limb

prostheses, suspension with an Icelandic Roll-On Silicone Socket

(ICEROSS) system was preferred by the amputees because of better

suspension, fit, stump protection, and comfortwhen comparedwith the

other suspension methods (Hachisuka et al., 1998; Heim et al., 1997).

The function of the prosthesis was also improved with silicone liners

when compared to the other suspension systems (Baars and Geertzen,

2005; Cluitmans et al., 1994; Legro et al., 1999; Trieb et al., 1999).

Prosthetic suspension and fit are said to be correlated to pistoning

(Commean et al., 1997; Grevsten, 1978; Newton et al., 1988; Sanders

et al., 2006). Thus, measuring the pistoning within the socket would be

helpful in determiningthe optimal prosthetic fit (Commean et al., 1997).

Liner technology has evolved significantly and many liners with

different properties are available today (Sanders et al., 2004). Clinicians

often try to choose appropriate liners (soft socket) for each subject

based on their personal experience and producers' technical information

(Klute et al., 2010; McCurdie et al., 1997). Silicon liners were

introduced in 1986 and their main advantage was claimed to be

enhanced bond with the stump and therefore, better suspension

compared with the other soft sockets (Baars et al., 2008). Silicon liners

are said to reduce pistoning of the stump and the bone compared with

the polyethylene foam (Pelite) liners (Narita et al., 1997; Söderberg

et al., 2003; Yigiter et al., 2002). It has been showed either clinically or by 
questionnaire. A clinical study by Tanner and Berke (2001) found only

$2 \mathrm{~mm}$ of pistoning of the residual limb with silicone liner and shuttle

lock inside the TSB socket,while Sanders et al. (2006) stated the amount

of pistoning of $41.7 \mathrm{~mm}$ with PTB socket. Questionnaire study by

Cluitmans et al. (1994), Hachisuka et al. (1998) and Datta et al. (1996) reported improved suspension in 96, 63 and $15 \%$ of their subjects with the silicon liners, respectively.

Manufacturers of prosthetic components have always attempted to

come up with new innovative suspension systems to lessen pistoning

(Trieb et al., 1999; Wirta et al., 1990). The recent development of the

prosthetic liner Seal-In® X5 byÖssur (Reykjavik, Iceland), a newsuction

suspension liner with hypobaric sealing membrane around the silicon

liner without an external sleeve or shuttle lock which increases surface

contact with the socket wall, motivated us to study the effects of this

newliner on prosthetic suspension. Furthermore, the manufacturer has

claimed that the Seal-In® X5 andDermo® Liner can reduce the pistoning

during ambulation (Össur, 2008). The objective of this study, therefore,

was to compare the effects of the new Seal-In® X5 Liner and Dermo®

Liner (both are considered silicone liners; Fig. 1) on transtibial

prosthetic pistoning. The comparison was performed in full-weight

bearing, semi-weight bearing, and non-weight bearing onthe prosthetic

limb, and also under three static vertical loading conditions $(30 \mathrm{~N}, 60 \mathrm{~N}$,

and 90 N) using the Vicon Motion System.

In the literature review, as far as authors are aware, no study

regarding the effects of Seal-In® X5 and Iceross Dermo® Liners on

transtibial prosthetic suspensionwas found. Fewstudies that compared

other suspension systems used techniques other than ours to monitor

pistoning actionwithin the transtibial or transfemoral socket. A number

of methods, such as the ultrasonicmethod (Convery and Murray, 2000), 

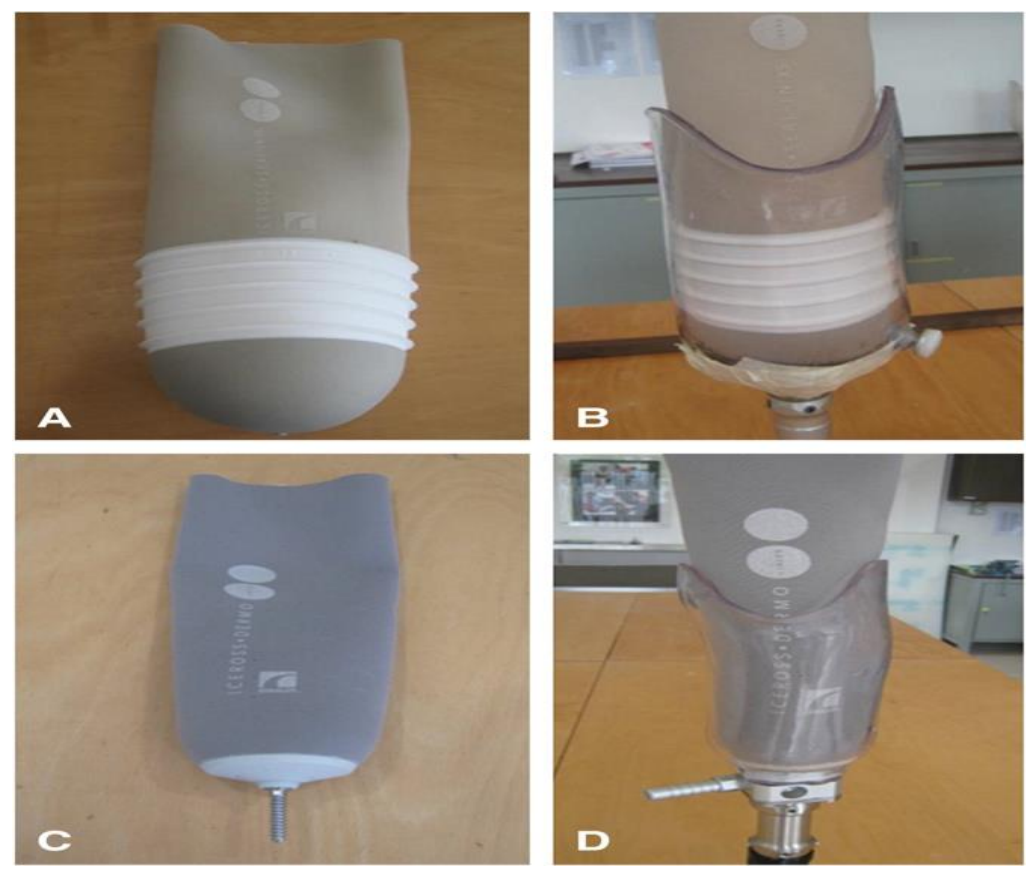

Fig. 1. Transtibial suspension systems used in this study (A) Seal-In® X5 Liner;

(B) transparent socket and valve; (C) Dermo $₫$ Liner; (D) transparent socket and shuttle lock.

roentgenological method (Erikson and Lemperg, 1969; Grevsten and Erikson, 1975; Söderberg et al., 2003), X-ray and cineradiography (Lilja et al., 1993; Narita et al., 1997), or spiral computerized tomography (CT) (Madsen et al., 2000) have been used to measure either the bony structures' positions within the stump relative to the socket or residual limb slippagewithin the socket. Photoelectric sensors and custommade transducers have been also used (Abu Osman et al., 2010a; Abu Osman et al., 2010b; Sanders et al., 2006). But, since these methods are costly and X-ray could be harmful to the subjects' bodies, these studies have been mostly conducted as case studies in laboratories. Studying pistoning with the Vicon Motion System was employed for the first time in this study.

\section{Methods}

Six male unilateral transtibial amputees with a mean age of 43 (SD 16.5) and mobility grade K2-K3, based on the American Academy of Orthotists \& Prosthetists, participated in this study on a voluntary basis. The mean time since amputation was 5 years. All subjects had undergone amputation at least 3 years before participating in the study. Ethical approval was granted from the University of Malaya Medical Centre (UMMC) Ethics Committee. All subjects were asked to provide a written informed consent. Characteristics per subject are listed in Table 1. 
The inclusion criteria were unilateral transtibial amputees with at

least $13 \mathrm{~cm}$ stump length (inferior edge of patella to distal end of the

stump), stable limb volume, intact upper limbs (hand strength), no

pain or wound in their stumps, and mobility without assistive devices,

such as cane.

First, two transtibial prostheses with similar feet (Flex-Foot

Talux $\left.{ }^{\circledR}\right)$ and two different liners, Iceross Dermo® Liner with shuttle

lock (Icelock-clutch 4 H214 L 214000) and Iceross Seal-In® X5

transtibial liner with valve (Icelock Expulsion Valve 551), were made

for each subject by a Registered Prosthetist and Orthotist.

All the prostheses were made by a single prosthetist to avoid

variability due to manufacture, fit, and alignment. All the subjects

were fitted with a transparent check socket to ensure that the socket

was Total Surface Bearing (TSB) (Staats and Lundt, 1987), and the

inside of the socket was visible. Then they were asked to walk with

their two new prostheses in the Brace and Limb laboratory

(Department of Biomedical Engineering, University of Malaya,

Malaysia) to become familiar with and adapt to the new liners and

Flex-Foot Talux® (Össur).

The prosthetist checked the alignment and fit of the prosthetic socket; then all the subjectswere given a trial period of at least 4 weeks to become accustomed to the new prostheses. Following this trial period, subjects attended the motion analysis laboratory formonitoring the pistoning within the socket by collecting data via a 7-camera Vicon 612 system (Oxford Metrics; Oxford, UK). Sixteen reflective markers according to the Helen Hayes marker set were attached to the subjects' prosthesis and sound lower limbs. On the prosthetic side, the knee and tibia markers were located on lateral proximal socket wall (LPS) and lateral distal end of the socket (LDS), respectively (Fig. 2). In order to measure the liner vertical movement two extra markers were attached to a) lateral liner below the knee joint (LLin1) and b) $5 \mathrm{~cm}$ below the

LLin1(LLin2). A pilot study showed that the knee flexion and extension can bias the real amount of pistoning and should be eliminated.

Therefore, in order to ensure the measurement accuracy the two extra markers (LLin1, 2) were attached over the liner below the knee level to avoid the kneemotion. Static trialswere carried out using deadweights. The trials were developed to ensure accurate application of loads in the vertical direction, held rigidly in a vertical attitude, and then loaded using weights hung from the prosthetic foot via wire. To simulate the centrifugal force during gait (Board et al., 2001; Commean et al., 1997; Narita et al., 1997), known loads $(30,60$, and $90 \mathrm{~N})$ were then applied to the prosthetic foot (Flex-Foot Talux $®$ ) and then unloaded (Fig. 3) while the signal outputswere recorded using the motion analysis system. The trials were repeated five times. Each subject was required to complete different static conditions such as single limb support on prosthetic limb (full-weight bearing), double limb support (semi-weight bearing), nonweight 
bearing (subjects suspended the prosthetic limb from the edge

of a table), and adding and removing the loads on the prosthetic limb. Each subject went through three different vertical loading conditions.

Using a transparent socket enabled us to locate markers on the liner inside the hard socket (two fine, paper-thin 2D markers were attached on the liner inside the hard socket) so that the cameras would detect the marker and we would be able to see the pistoning movement inside the socket (Fig. 2). Moreover, by locating the markers all on one segment, that is, the tibia we could avoid knee flexion and thereby any fake displacement. During the pilot trials we noticed that a transparent socket resulted in reflections that were detected as markers by the cameras; hence we covered the transparent socket wall with paper tape, except the areas to which we added two new markers.

For calculating pistoning within the socket, we used the distance between two markers (one marker on the liner (LLin1) and another one on the socket (LPS) during full-weight bearing on the prosthesis as a baseline. Then we compared the other conditions with the baseline to identify any pistoning movement. Additionally, an informal subjective subject survey and feedback was carried out to obtain qualitative information about the liners. Statistical data was analyzed with SPSS 17.0, and P-values of 0.05 or less were chosen to reflect statistical significance. Wilcoxson test was employed to compare the effect of two liners on the pistoning.

Results

The results obtained from static evaluation of Seal-In® X5 and Dermo® Liner showed that there was a significant difference between the two liners (Pb0.05). Pistoning between Seal-In® X5 and the socket was not the same as that with Iceross Dermo® Liner and socket $(71 \%$ less). The average displacement in the six subjects between the two liners and the socket under different static conditions (after adding loads and after removing loads) is listed in Table 2. The subjective feedback of the participants indicated less skin stretch, and more feeling of security (two amputees) with Seal-In®X5 Liner. However, diabetic subjects' main complaint was about donning and doffing the Seal-In $\otimes X 5$; and when they were asked to choose one liner, they chose Dermo® Liner. When the loads were added to the prosthesis the subjects felt more comfortable at the end of residual limb with the Seal-In®X5.

Full text is available at :

http://ac.els-cdn.com/S0268003311001859/1-s2.0-S0268003311001859-main.pdf? tid=82dd2720-a019-11e3952c-00000aab0f01\&acdnat $=1393551905$ 7c6f57e23f3b01c0a079afa004aac1b0 
http://www.ncbi.nlm.nih.gov/pubmed/21794965

http://www.sciencedirect.com/science/article/pii/S0268003311001859 